Ex, P.; Felgner, S.; Henschke, C.

\title{
Rechtsprechungen zur Kostenerstattung von neuen Gesundheitstechnologien in der GKV: Eine systematische Übersicht
}

Journal article | Accepted manuscript (Postprint)

This version is available at https://doi.org/10.14279/depositonce-8238

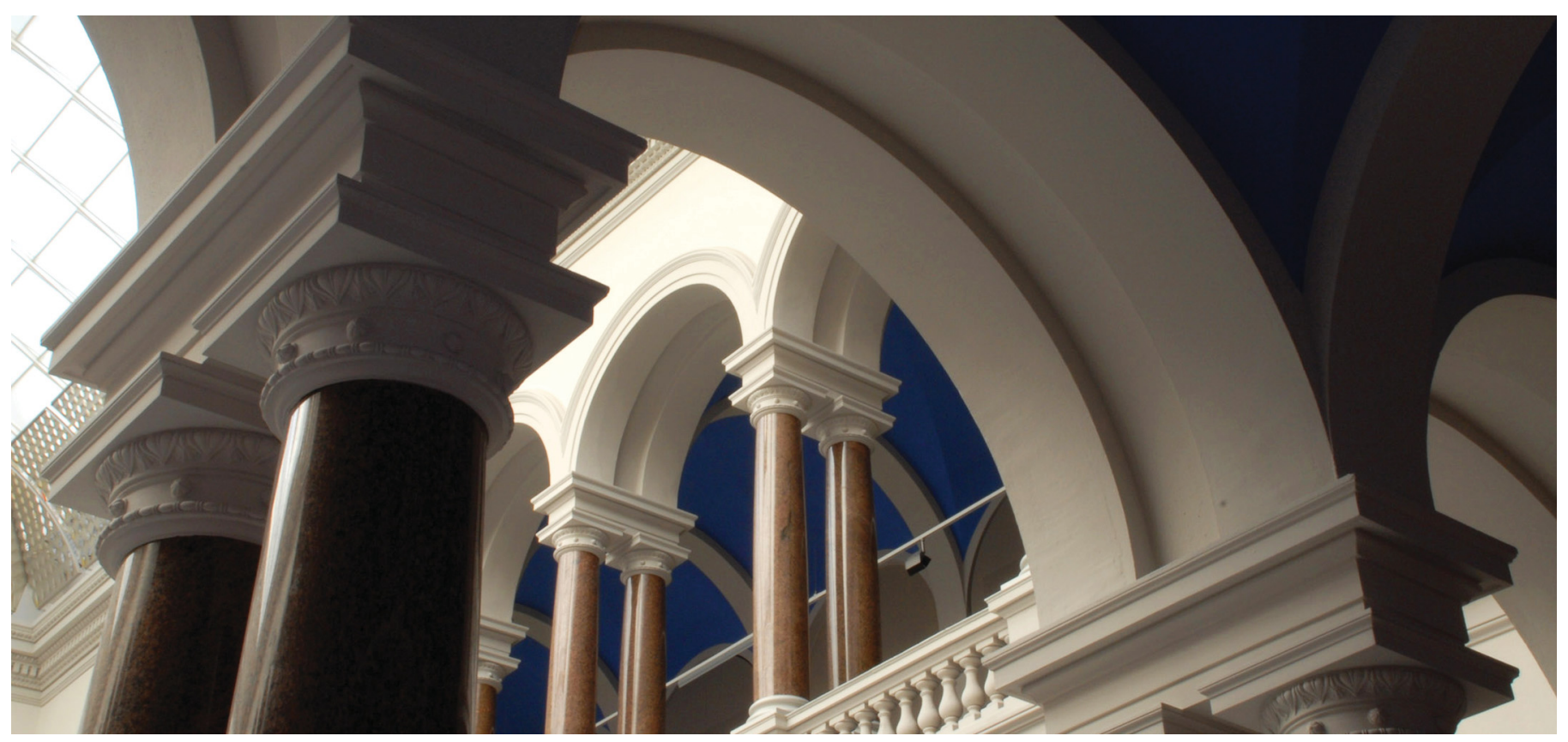

Ex, P., Felgner, S., \& Henschke, C. (2018). Rechtsprechungen zur Kostenerstattung von neuen Gesundheitstechnologien in der GKV: Eine systematische Übersicht. Zeitschrift für Evidenz, Fortbildung und Qualität im Gesundheitswesen, 131-132, 8-16. https://doi.org/10.1016/j.zefq.2017.12.004 
Patricia Ex, Susanne Felgner, Cornelia Henschke

Rechtsprechungen zur Kostenerstattung von neuen Gesundheitstechnologien in der GKV: Eine systematische Übersicht

Jurisdictions on the reimbursement of new medical technologies

by public health insurance: A systematic review 


\section{Zusammenfassung}

Hintergrund. Die Erstattung neuer Gesundheitstechnologien wird nicht selten über Sozialgerichte eingeklagt. Dabei liegt es nahe, dass gerichtliche Entscheidungen auch Einzelfallentscheidungen der Krankenkassen und damit den Patientenzugang insgesamt beeinflussen.

Ziel der Studie. Ziel ist anhand von Rechtsprechungen zu identifizieren, welche Technologien wiederholt eingeklagt wurden und ob diese zu einer Kostenerstattung führten. Dabei liegt der Fokus auf Unterschieden zwischen Sektoren, Technologiegruppen sowie Indikationen. Auf dieser Grundlage soll exemplarisch anhand von vier Technologien analysiert werden, ob die Ergebnisse der gerichtlichen Entscheidungen zur Kostenerstattung gleicher Gesundheitstechnologien über die Jahre variieren.

Methodik. Mittels einer systematischen Datenbankrecherche wurden Rechtsprechungen von deutschen Sozialgerichten zu neuen Gesundheitstechnologien der Jahre 2011 bis 2016 gesucht. Ausgewertet wurden solche Rechtsprechungen, bei denen Sozialgerichte über die Kostenerstattung von Technologien zur Behandlung einzelner Personen entschieden hatten.

Ergebnisse. Die Recherche brachte 284 relevante Rechtsprechungen hervor. Bei einem Drittel der Klagen wurden die Krankenkassen zur Kostenerstattung verpflichtet, im stationären Sektor häufiger als im ambulanten. Technologien zu Erkrankungen der Augen und Ohren wurden am häufigsten bewilligt. Dabei kamen Sozialgerichte auch zu unterschiedlichen Kostenerstattungsentscheidungen bei inhaltlich und zeitlich ähnlichen Fällen. Die Rechtsprechungen stehen in Teilen im Widerspruch zu nachfolgenden Entscheidungen der Methodenbewertung durch den Gemeinsamen Bundesausschuss (G-BA).

Schlussfolgerung. Die Uneinheitlichkeit der Erstattungsentscheidungen kann zu Unsicherheit darüber führen, welche Technologien den ,allgemein anerkannten Stand der 
medizinischen Erkenntnisse‘ erfüllen. Dabei erweist es sich für Patienten und Ärzte als problematisch, dass Klagen zu Behandlungsmethoden über Jahre hinweg eingereicht werden, ohne dass Nutzen und Erstattungsfähigkeit systematisch bewertet sind.

\section{Schlüsselwörter}

Neue Untersuchungs- und Behandlungsmethoden, neue Gesundheitstechnologien, Rechtsprechung Sozialgerichte, Verbotsvorbehalt, Nikolausurteil, Systematische Übersicht 


\section{Abstract}

Background. Legal actions are taken at social courts to receive reimbursement for new medical technologies in Germany. It is likely that these judicial decisions also affect sickness funds' decisions on requests for reimbursement and thus patient access to new technologies in general.

Aims of the study. The aim of this study is to identify what technologies are sued for, and which lead to reimbursement. The focus is on differences between sectors, technology groups and indications. Based on this, we analyse in a case study whether judicial decisions on the reimbursement of the same technologies vary across the years.

Material and Methods. Based on a systematic review, we identified judicial decisions of German social courts on new technologies for the years 2011 to 2016 . Those judicial decisions were included in the analysis that decided on the reimbursement of a technology for treating individual persons.

Results. We considered 284 judicial decisions on new technologies in the analysis. One third of them had to be reimbursed by sickness funds, with a higher percentage in inpatient than in outpatient care. Technologies for diseases of eyes and ears were granted most frequently. Judicial decisions on the same technology at the similar point in time differ in their results regarding cost reimbursement.

Conclusions. The decision whether a new technology is reimbursed seems not to follow a systematic approach. In the context of the seemingly innovation friendly policy in inpatient care, there is uncertainty with regards to the 'generally accepted state of medical knowledge.' It is problematic for patients and for treating physicians that legal actions are taken for the same technologies over many years without undergoing a systematic assessment of their benefit. 


\section{Keywords}

'New Diagnostic and Treatment Methods,' new health technologies, jurisdiction of the Social Courts, 'permission with the reservation of prohibition, ' jurisdiction of St. Nicholas' day, systematic review 


\section{Einleitung}

Die Kostenerstattung von neuen Gesundheitstechnologien durch die gesetzliche Krankenversicherung (GKV) erfolgt generell, wenn diese in den Leistungskatalog aufgenommen sind. Trotzdem gibt es nicht selten Fälle, über die per Einzelfallantrag an eine Krankenkasse entschieden wird (§ 13 Abs. 3 SGB V). Ein Beispiel sind einige Leistungen im stationären Sektor: Gemäß dem Prinzip des „Erlaubnis mit Verbotsvorbehalt“ dürfen Methoden im stationären Sektor zulasten der GKV angewandt werden, wenn sie nicht explizit vom Gemeinsamen Bundesausschuss (G-BA) ausgeschlossen wurden ( $§ 137 \mathrm{c}$ SGB V). Ist die Leistung nicht teurer als ihre Alternativen, erfolgt die Finanzierung über das Fallpauschalensystem. Ansonsten wird ein Einzelfallantrag an die Krankenkasse gestellt oder ein Innovationsentgelt beantragt. Im ambulanten Sektor hingegen gilt das Prinzip „Verbot mit Erlaubnisvorbehalt“, nach dem nur solche Leistungen erstattet werden, die vorab vom G-BA positiv beschieden wurden (§ 135 SGB V). Doch auch hier sind Einzelfallanträge an die Krankenkassen möglich. Weitere Gründe für Einzelfallanträge sind Arzneimittel außerhalb ihres zugelassenen Anwendungsgebietes, im sog. „Off-Label-Use“ und Leistungen nach dem Nikolausurteil des Bundesverfassungsgerichts (BVerfG). So beschied das BVerfG am 06.12.2005, dass Patienten mit einer lebensbedrohlichen oder regelmäßig tödlich verlaufenden Krankheit, für die schulmedizinische Behandlungsmethoden nicht vorliegen, Anspruch auf alle Methoden haben, die eine „nicht ganz entfernt liegende Aussicht auf Heilung oder auf eine spürbare positive Einwirkung auf den Krankheitsverlauf“" (Az. 1 BvR 347/98) versprechen (sog. „Nikolausurteil“). Mit dem GKV-Versorgungsstrukturgesetz wurde diese Auffassung zum 01.01.2012 in das Sozialgesetzbuch aufgenommen (§ 2 Abs. 1a SGB V). Mit diesen Ansätzen soll sichergestellt werden, dass Patienten auch Zugang zu Leistungen erhalten, die (noch) nicht im Leistungskatalog der GKV enthalten, im Rahmen der Behandlung jedoch notwendig sind.

Um erstattet werden zu können, muss eine Leistung die Maßgabe erfüllen, dass Qualität und Wirksamkeit dem „allgemein anerkannten Stand der medizinischen Erkenntnisse“ 
entsprechen und den „medizinischen Fortschritt“ berücksichtigen (§ 2 Abs. 1 SGB V). Auch müssen sie aus Verantwortung für das Solidarsystem „ausreichend, zweckmäßig und wirtschaftlich“ sein und dürfen „das Maß des Notwendigen nicht überschreiten“ (§ 12 SGB V). Krankenkassen sind zur Übernahme der Leistungen verpflichtet, wenn die Behandlung notwendig ist, um „Krankheiten zu erkennen, zu heilen, ihre Verschlimmerung zu verhüten oder Krankheitsbeschwerden zu lindern“ (§ 27 SGB V). Die Erstattungspflicht von neuen Gesundheitstechnologien ist damit in einigen Fällen nicht eindeutig geklärt. Individuelle Patienten, oft unterstützt von behandelnden Ärzten, haben tendenziell ein Interesse daran, Leistungen zu erhalten, die ihren Gesundheitszustand verbessern oder dies zumindest in Aussicht stellen. Die Erwartung ist dementsprechend, dass innen diese Leistungen durch die Krankenkassen finanziert werden. Im Sinne der Solidargemeinschaft ist jedoch eher eine Finanzierung von nachweislich funktionierenden Ansätzen sinnvoll. Ärzte möchten eher auf ein breites (wirksames) Leistungsspektrum zurückgreifen, da sie aus medizinischer Sicht über die Notwendigkeit einer Leistung für den Patienten entscheiden, während der Fokus von Krankenkassen zusätzlich auf dem Wirtschaftlichkeitsaspekt liegt. Zudem sind Wirksamkeitsnachweise von neuen Medizinprodukten meist nicht ausreichend erbracht, da für die europäische Marktzulassung Nachweise der Sicherheit und technischen Leistungsfähigkeit vorzulegen sind [1]. Basierend auf diesen unterschiedlichen Interessen sowie der Frage nach der Notwendigkeit einer Leistung, besteht oftmals Uneinigkeit über die Erstattungspflicht zwischen Patienten, Ärzten und Krankenkassen.

Vor diesem Hintergrund sowie den allgemein formulierten Regelungen im Sozialgesetzbuch führen die verschiedenen Interessenslagen zu Klagen an deutschen Sozialgerichten, die für Rechtsstreitigkeiten der GKV zuständig sind. Eine Klage wird dabei zunächst vom Sozialgericht entschieden und - im Falle einer Berufung bzw. Revision - an das jeweilige Landessozialgericht und ggf. anschließend an das Bundessozialgericht übergeben. Beim Bundesverfassungsgericht können, bei der Verletzung von Grundrechten, Klagen aller Gerichtsbarkeiten der obersten Instanz als 
Verfassungsbeschwerde eingereicht werden. Patienten, die eine Behandlung einklagen, haben zunächst Einzelfallanträge bei ihrer Krankenkasse gestellt (ggf. wurde ein Gutachten des Medizinischen Dienstes der Krankenkassen erstellt), dem Ablehnungsbescheid widersprochen, i. d. R. anschließend Klage beim Sozialgericht eingereicht und meist mehrere Instanzen durchlaufen.

Entscheidungen des G-BA sowie die Anpassung des Leistungskatalogs wurden national und international bereits mehrfach in wissenschaftlichen Studien untersucht [2-6]. Die Erstattungsentscheidungen für Leistungen, die auf Basis von Einzelfallentscheidungen der Sozialgerichte getroffen wurden, wurden bislang nicht untersucht.

Ziel dieser Studie ist es, in einem ersten Schritt auf Grundlage der Rechtsprechungen deutscher Sozialgerichte in einer Querschnittsanalyse herauszustellen, für welche Behandlungen Klagen vorrangig gestellt werden und welche zu einer Kostenerstattungspflicht der jeweiligen Krankenkasse führen. In einem zweiten Schritt soll im Rahmen einer Längsschnittanalyse beispielhaft für einige Technologien untersucht werden, ob das Ergebnis von Entscheidungen bzgl. der Anwendung und Erstattung einer Technologie über die Jahre variiert. Dabei werden die Beschlüsse des G-BA den juristischen Entscheidungen der einzelnen Technologien gegenübergestellt. In der sozialrechtlichen Debatte und vom G-BA wird zumeist der Begriff „Neue Untersuchungs- und Behandlungsmethode" verwendet, also eine Methode mit eigenem theoretisch-wissenschaftlichem Konzept [7]. Da diese Anforderung in den Rechtsprechungen des Datensatzes nicht überprüft wird, verwenden wir im Folgenden den im internationalen Diskurs gängigen Begriff „neue Gesundheitstechnologie“ (new health technology), der Arzneimittel, Medizinprodukte, ebenso wie Untersuchungs- und Behandlungsmethoden (international als Prozeduren, engl.: procedures, bezeichnet) umfasst [8-9]. Als „neu“ gelten sie, solange sie nicht im Leistungskatalog der GKV gelistet sind [6]. 


\section{Material und Methodik}

Die Studie basiert auf einer systematischen Recherche von Rechtsprechungen deutscher Sozialgerichte zu neuen Gesundheitstechnologien. Da systematische Übersichtsarbeiten in den Rechtswissenschaften nicht verbreitet sind, wurden die Methoden zur Erstellung systematischer Übersichtsarbeiten des Centre for Reviews and Dissemination sowie des Instituts für Qualität und Wirtschaftlichkeit im Gesundheitswesen (IQWiG) von gesundheitswissenschaftlichen Studien auf Rechtsprechungen übertragen [10,11]. Die Recherche erfolgte in der Datenbank juris mittels der Suchbegriffe „Neue Untersuchungsund Behandlungsmethode“, „Neue Untersuchungsmethode“, „Neue Behandlungsmethode“, „NUB“ und „Innovation“ in Kombination mit dem Suchbegriff „SGB V“ für die gleichnamige Norm sowie mittels der Begriffe „SGB V § 137c“ und „SGB V§ 135“ in mehreren Teilrecherchen (s. Abbildung 1). Für die Suche wurde der Begriff „neue Untersuchung- und Behandlungsmethode“ verwendet, da dieser im deutschen Sozialrecht einschlägig ist. Da inhaltlich relevante Rechtsprechungen von den Gerichten direkt zur Publikation an die Datenbank gegeben werden, ist davon auszugehen, dass die Datenbank juris alle für diese Fragestellung inhaltlich relevanten Urteile und Beschlüsse enthält. Zudem gibt es keine Alternative zur dieser Datenbank, deren zusätzliche Verwendung einen Mehrwert brächte. Bei den Suchbegriffen wurden neben dem Wortstamm auch ihre Variationen recherchiert. Es wurden ausschließlich Rechtsprechungen mit Gerichtsentscheidung zwischen dem 01.01.2011 und dem 01.08.2016 gesucht, mit letzter Aktualisierung der Recherche am 26.08.2016.

Alle potentiell relevanten Rechtsprechungen wurden im Volltext gesichtet und nach folgenden gestuften Kriterien ausgeschlossen:

1. Rechtsprechung nicht von deutscher Sozialgerichtsbarkeit ${ }^{1}$,

2. Rechtsprechung höherer Instanz vorhanden²,

1 Rechtsprechungen des BVerfG werden in der Analyse berücksichtigt, wenn die Klage vorab in der Sozialgerichtsbarkeit beschieden wurde. 
3. keine Krankenbehandlung einer Person oder mehrerer konkreter Personen,

4. keine Anwendung einer Technologie und

5. Klage nicht zu Kostenerstattung von Leistungen.

In systematischen Übersichtsarbeiten werden üblicherweise Qualitätsbewertungen der Literatur durchgeführt [10], bei denen die einzuschließenden Studien auf methodische Minimalstandards überprüft werden. Bei der Übertragung der methodischen Vorgehensweise auf Rechtsprechungen ist davon auszugehen, dass alle rechtskräftigen Entscheidungen der deutschen Gerichte keiner Qualitätsbewertung unterzogen werden müssen, sondern per se in die Analyse einbezogen werden können.

Aus den relevanten Rechtsprechungen wurden folgende Variablen extrahiert: (1) die Instanz, von der die Klage entschieden wurde, d. h. Sozialgericht (SG), Landessozialgericht (LSG), Bundessozialgericht (BSG) oder BVerfG, (2) das Bundesland in dem die Klage erstinstanzlich eingereicht worden war, (3) das Entscheidungsdatum der Rechtsprechung, (4) die Art der Technologie (,Technologiegruppe'), (5) die Indikation, (6) Kläger und Beklagter, z. B. Patient oder Krankenhaus und Krankenkasse, (7) der Sektor der Leistungserbringung (ambulant / stationär), (8) der vom Kläger geforderte Erstattungsbetrag der Technologie und (9) die Entscheidung zur Erstattungsverpflichtung, d. h. ob infolge der Klage die Kosten der Technologie von der Krankenkasse erstattet werden mussten.

Die Variable ,Technologiegruppe‘ wurde in die gängigen Kategorien Medizinprodukte, Arzneimittel und Untersuchungs- und Behandlungsmethoden (Prozeduren) unterteilt, wobei Hilfsmittel als Teil von Medizinprodukten zusätzlich abgebildet wurden. Grund hierfür ist, dass Hilfsmittel sich von anderen Medizinprodukten unterscheiden: Patienten wenden Hilfsmittel eigenständig an und auch die Regelungen zur Erstattung von Hilfsmitteln sind unterschiedlich im Vergleich zu anderen Medizinprodukten [12]. Um

\footnotetext{
${ }^{2}$ Im Beschluss einer Rechtsprechung sind, sofern vorhanden, die Rechtsprechungen niedrigerer Instanzen dieses Falles vermerkt. In der Datenbank konnten damit alle Rechtsprechungen identifiziert werden, für die eine Rechtsprechung höherer Instanz vorlag.
} 
wesentliche Anwendungsgebiete herauszustellen, erfolgte eine Gruppierung der Klagen nach Indikationen. Um mit möglichst wenigen Indikationsgruppen einheitliche Kategorien zu bilden, wurde im Gegensatz zu den anderen Variablen, die vorab definiert worden waren, hierbei nicht auf bestehende Kategorien (z. B. ICD-10 als statistische Klassifikation von Krankheiten) zurückgegriffen. Die Indikationsgruppen wurden bei Durchsicht der eingeschlossenen Rechtsprechungen induktiv erstellt. Dabei wurden Indikationsgruppen gebildet, sofern es mindestens drei Fälle an Klagen gab. Alle Indikationsgruppen mit weniger als drei Fällen wurden in die Kategorie „Sonstiges“ subsumiert. Die gebildeten Gruppen werden in den Ergebnissen aufgezeigt.

Im ersten Teil der Analyse wurden die Gesundheitstechnologien extrahiert, die Gegenstand der Rechtsprechungen waren. Die Rechtsprechungen wurden im Folgenden hinsichtlich der Erstattungsverpflichtungen nach Sektor der Leistungserbringung und nach Technologiegruppe sowie nach Indikationsgruppen analysiert. Auch die von den Klägern geforderte Erstattungshöhe wurde dabei berücksichtigt. Die systematische Recherche, die Sichtung und Selektion der Fälle nach den definierten Ausschlusskriterien sowie die Extraktion der Variablen wurden von zwei Autoren parallel durchgeführt. Unstimmigkeiten wurden unter Einschluss einer dritten Person per Konsens entschieden. Im zweiten Teil der Analyse wurde die Variabilität der Erstattungsentscheidungen über die Jahre untersucht, sowie den Beschlüssen des G-BA gegenübergestellt. Um für den Vergleich der Entscheidungen eine ausreichende Fallzahl an Rechtsprechungen pro Technologie sicherzustellen, wurde dieser Teil der Analyse für die vier am häufigsten beklagten Technologien exemplarisch durchgeführt.

\section{Ergebnisse}

Ergebnisse der systematischen Recherche und des Selektionsprozesses

Über die Suche in der Datenbank juris wurden insgesamt 955 Treffer identifiziert. Durch die Suche mit inhaltlich ähnlichen Suchbegriffen in mehreren Teilrecherchen brachte die Recherche einzelne Rechtsprechungen mehrfach hervor. Dementsprechend wurde die 
Gesamtheit der Treffer zuerst von Duplikaten bereinigt. Nach ihrem Ausschluss

verblieben 408 potentiell relevante Rechtsprechungen. Abbildung 1 gibt als

Flussdiagramm eine Übersicht der Ergebnisse der systematischen Recherche und stellt den Selektionsprozess gemäß den Ausschlusskriterien dar.

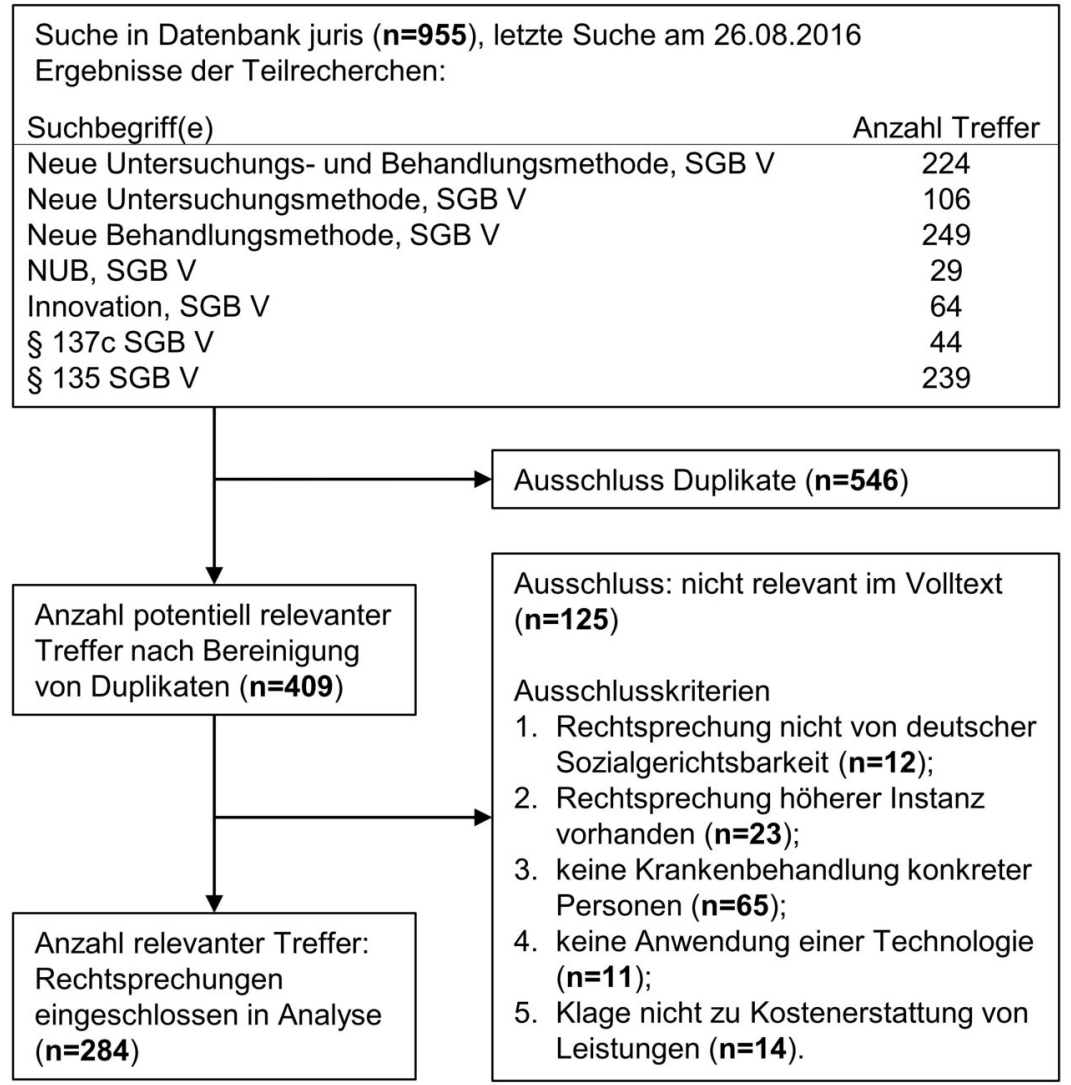

Abbildung 1. PRISMA-Diagramm: Ergebnisse der Recherche und des Selektionsprozesses (nach dem Modell des Centre for Reviews and Dissemination sowie des Institutions für Qualität und Wirtschaftlichkeit im Gesundheitswesen)

Es folgte die Sichtung der Rechtsprechungen in ihrem Volltext sowie deren Selektion gemäß den festgelegten Kriterien (vgl. Ausführungen zu Material und Methodik). Insgesamt wurden hierbei 125 Rechtsprechungen ausgeschlossen, da sie mindestens ein Ausschlusskriterium erfüllten: Zwölf Klagen richteten sich in erster Instanz nicht an ein Sozialgericht ${ }^{3}$, für 23 Rechtsprechungen lagen Entscheidungen höherer Instanz zum gleichen Fall vor. In 65 Rechtsprechungen war nicht die Behandlung einer oder mehrerer

${ }^{3}$ Durch die Eingrenzung auf Sozialgerichte sind nur Fälle aus der GKV enthalten, da für Rechtsstreitigkeiten der Privaten Krankenversicherung (PKV) die Amts- und Landgerichte zuständig sind. 
konkreter Personen Klagegegenstand, sondern z. B. die Erbringung einer Behandlung für eine allgemeine Gruppe von Patienten. Das Kriterium „keine Anwendung einer Technologie" traf auf elf Rechtsprechungen zu. Für weitere 14 Rechtsprechungen war der Gegenstand der Klage nicht die Kostenerstattung von Leistungen, sondern z. B. der Sektor der Leistungserbringung. Nach Ausschluss der Duplikate und der Rechtsprechungen gemäß Ausschlusskriterien gingen 284 Rechtsprechungen in die Analyse ein.

\section{Ergebnisse der Analyse}

Von den in die Analyse eingeschlossenen Klagen betreffen 60 (21\%) eine Behandlung im stationären und 224 (79\%) eine Behandlung im ambulanten Sektor. Die Mehrheit der Klagen (56\%) wurde auf zweiter Instanz nach einer Revision bzw. Berufung von einem LSG beschieden, 33 Prozent auf erster Instanz. Knapp zehn Prozent der Klagen wurden bis zum BSG fortgeführt und ein Prozent vom BVerfG entschieden.

Eine Betrachtung über die Kalenderjahre zeigt, dass die Anzahl der Rechtsprechungen jährlich zunahm, mit 45 Urteilen im Datensatz für das Jahr 2011 stetig ansteigend bis 66 Urteile im Jahr $2015^{4}$. Das entspricht einer Zunahme von 47 Prozent. Die Daten suggerieren, dass pro Bundesland unterschiedlich viele Klagen beschieden wurden. Gewichtet auf die durchschnittliche Einwohnerzahl pro Bundesland von 2011 bis $2015^{5}$ und unter der Annahme einer Gleichverteilung des GKV-versicherten Personenkreises wurde mit 0,74 Rechtsprechungen pro 100.000 Einwohner die höchste Klagerate in Hamburg in dem betrachteten Zeitraum von fünf Jahren beschieden. Die niedrigste Rate ist mit 0,15 Rechtsprechungen pro 100.000 Einwohner in Bayern gegeben.

Eine Betrachtung von Klägern und Beklagten zeigt folgendes Bild: Die überwiegende Mehrheit der Klagen wurde zwischen Patienten (oder deren Rechtsnachfolge) als Kläger

${ }^{4} 2016$ wurde hierbei nicht berücksichtigt, da die Suche nur Rechtsprechungen bis zum 01.08.2016 beinhaltet.

${ }_{5}$ Da die Bundesländer Berlin und Brandenburg sowie Bremen und Niedersachen jeweils ein Landessozialgericht unterhalten, gibt es insgesamt 14 Landessozialgerichte. Die Daten zu Bevölkerungszahlen der Bundesländer sind vom Statistischen Bundesamt mit Datenstand vom 31.12.2011, 2012, 2013, 2014 und 2015 (siehe URL: https://www-genesis.destatis.de/genesis/online/link/tabellen/12411*, letzter Zugriff am 18.10.2016). 
und Krankenkassen als Beklagten verhandelt (94\%). In fünf Prozent der Rechtsprechungen im Datensatz verklagt ein Leistungserbringer (i. d. R. ein Krankenhaus) eine Krankenkasse. Die Konstellation, dass eine Krankenkasse ein Krankenhaus verklagt, kam in einem Fall zur Anwendung.

\section{Erstattungsverpflichtung nach Sektor und Technologiegruppe}

Tabelle 1 gibt eine Übersicht über die Erstattungsverpflichtungen der Krankenkassen, aufgeschlüsselt nach Technologiegruppe und Sektor der Leistungserbringung. In 35 Prozent $(n=98)$ aller Fälle wurden die Krankenkassen zur Erstattung verpflichtet. In fünf Fällen wies das BSG die Klage zurück an das zuständige Landessozialgericht und fällte keine Entscheidung zur Kostenerstattung. Dabei ist der Anteil der Erstattungsverpflichtungen im stationären Bereich mit 40 Prozent höher als im ambulanten (33\%). Im stationären Sektor fielen 48 der insgesamt 60 Rechtsprechungen in die Technologiegruppe der Untersuchungs- und Behandlungsmethoden; für 17 dieser Fälle wurde eine Erstattungsverpflichtung festgelegt. Im ambulanten Sektor mussten Arzneimittel und Hilfsmittel prozentual am häufigsten erstattet werden, Behandlungsmethoden deutlich seltener. Von den sieben Rechtsprechungen zu Medizinprodukten, die keine Hilfsmittel sind, betreffen alle den ambulanten Bereich. Von diesen wurde keine Leistung als erstattungspflichtig beschieden. Auch bei den Arzneimitteln wurden deutlich mehr Klagen für die Anwendung im ambulanten $(n=31)$ als im stationären Sektor $(n=9)$ eingereicht. Für den stationären Sektor führten diese jedoch prozentual häufiger zu einer Erstattungsentscheidung, nämlich in sechs von neun Fällen. Ein häufiger Klagegrund ist dabei die fehlende Erstattungsfähigkeit eines Arzneimittels bei Anwendung im Off-Label-Use. 
Tabelle 1

Erstattungsverpflichtung neuer Gesundheitstechnologien in Rechtsprechungen je Technologiegruppe, getrennt nach Sektoren

\begin{tabular}{|c|c|c|c|c|c|c|c|}
\hline & \multicolumn{3}{|c|}{ ambulant $(n=224)$} & \multicolumn{3}{|c|}{ stationär $(n=60)$} \\
\hline & & $\begin{array}{l}\text { Leistung } \\
\text { nicht zu } \\
\text { erstatten }\end{array}$ & $\begin{array}{l}\text { Leistung } \\
\text { zu } \\
\text { erstatten }\end{array}$ & $\begin{array}{c}\text { Klage } \\
\text { zurück- } \\
\text { verwiesen }\end{array}$ & $\begin{array}{l}\text { Leistung } \\
\text { nicht zu } \\
\text { erstatten }\end{array}$ & $\begin{array}{l}\text { Leistung } \\
\text { zu } \\
\text { erstatten }\end{array}$ & $\begin{array}{c}\text { Klage } \\
\text { zurück- } \\
\text { verwiesen }\end{array}$ \\
\hline \multirow{5}{*}{ 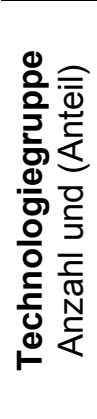 } & $\begin{array}{l}\text { Medizinprodukte }{ }^{6} \\
(n=100)\end{array}$ & $\begin{array}{c}54 \\
(55,67)\end{array}$ & $\begin{array}{c}40 \\
(41,24)\end{array}$ & $\begin{array}{c}3 \\
(3,09)\end{array}$ & $\begin{array}{c}2 \\
(66,67)\end{array}$ & $\begin{array}{c}1 \\
(33,33)\end{array}$ & 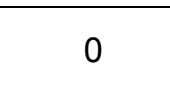 \\
\hline & $\begin{array}{c}\text { davon Hilfsmittel } \\
(n=89)\end{array}$ & $\begin{array}{c}47 \\
(52,81)\end{array}$ & $\begin{array}{c}40 \\
(44,94)\end{array}$ & $\begin{array}{c}2 \\
(2,25)\end{array}$ & 0 & 0 & 0 \\
\hline & $\begin{array}{l}\text { Arzneimittel } \\
\quad(n=40)\end{array}$ & $\begin{array}{c}17 \\
(54,84)\end{array}$ & $\begin{array}{c}14 \\
(45,16)\end{array}$ & 0 & $\begin{array}{c}3 \\
(33,33)\end{array}$ & $\begin{array}{c}6 \\
(66,67)\end{array}$ & 0 \\
\hline & $\begin{array}{c}\text { Behandlungs- } \\
\text { methoden }(n=144)\end{array}$ & $\begin{array}{c}76 \\
(79,12)\end{array}$ & $\begin{array}{c}20 \\
(20,83)\end{array}$ & 0 & $\begin{array}{c}29 \\
(60,42)\end{array}$ & $\begin{array}{c}17 \\
(35,42)\end{array}$ & $\begin{array}{c}2 \\
(4,17)\end{array}$ \\
\hline & Gesamt & $\begin{array}{c}147 \\
(65,63)\end{array}$ & $\begin{array}{c}74 \\
(33,04)\end{array}$ & $\begin{array}{c}3 \\
(1,34)\end{array}$ & $\begin{array}{c}34 \\
(56,67)\end{array}$ & $\begin{array}{c}24 \\
(40,00)\end{array}$ & $\begin{array}{c}2 \\
(3,33)\end{array}$ \\
\hline
\end{tabular}

Eine Aufgliederung der Fälle nach Technologiegruppen ohne Aufgliederung nach Sektoren zeigt, dass Arzneimittel in 50 Prozent der Rechtsprechungen zu diesem Bereich erstattet werden mussten, prozentual die höchste Erstattungsrate aller Technologiegruppen. In den Rechtsprechungen zu Medizinprodukten erhielten 41 Prozent eine Erstattungsverpflichtung, gefolgt von 26 Prozent der Klagen zu Behandlungsmethoden.

\section{Erstattungsverpflichtung nach Indikationsgruppen}

Die in den Rechtsprechungen verhandelten Technologien entsprachen den folgenden Indikationsgruppen: Tumorerkrankungen, Adipositas, Erkrankungen der Augen und Ohren, Erkrankungen des Bewegungsapparates, Erkrankungen des Mund- und Rachenraumes, Künstliche Befruchtung, Psychische Erkrankungen, Diabetes, Weitere chronische Erkrankungen, Schädeldeformitäten bei Säuglingen und 'Sonstiges'. Die Indikationsgruppe ,Weitere chronische Erkrankungen‘ beinhaltet beispielsweise Behandlungen bei Schlafapnoe und chronischem Schmerz. ,Sonstiges‘ bezieht sich auf Erkrankungen mit weniger als drei Rechtsprechungen pro Indikation. Ein Großteil der Tumore in der Indikationsgruppe Tumorerkrankungen war metastasierend; es war keine

\footnotetext{
${ }^{6}$ In der Kategorie Medizinprodukte ist in einem Fall ein Transplantat menschlichen Ursprungs enthalten.
} 
Häufung einer bestimmten Tumorerkrankung festzustellen. Technologien für Augen und

Ohren waren u. a. Hörgeräte und die Implantation von Linsen. Technologien zum

Bewegungsapparat umfassten vorrangig Prothesen. Der von den Klägern geforderte

Erstattungsbetrag für die Technologien variiert zwischen 66,78 Euro [13] und 293.792,20

Euro [14]; Tabelle 2 gibt einen Überblick über die Höhe der zu entscheidenden

Erstattungsbeträge pro Technologiegruppe. Dabei konnten nur die Rechtsprechungen einbezogen werden, bei denen die Höhe des geforderten Erstattungspreises in der Rechtsprechung genannt wurde $(n=210)$. Sofern in der Rechtsprechung festgestellt wurde, dass die Zahlungen dauerhaft zu leisten sind, beziehen sich die ermittelten Erstattungspreise auf Jahrestherapiekosten.

\section{Tabelle 2}

Von Klägern geforderter Preis der Technologie in Euro, gegliedert nach Technologiegruppe

\begin{tabular}{|c|c|c|c|c|c|}
\hline & & \multicolumn{4}{|c|}{ Preise der Technologien (in Euro) } \\
\hline & & Mittelwert & Median & Min. & Max. \\
\hline \multirow{5}{*}{ 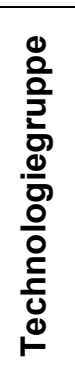 } & $\begin{array}{c}\text { Medizinprodukte } \\
(\mathrm{n}=89)\end{array}$ & $6.812,69$ & $2.526,50$ & 150 & 56.361 .32 \\
\hline & $\begin{array}{c}\text { davon Hilfsmittel } \\
(n=75)\end{array}$ & $7.051,46$ & $2.402,46$ & 150 & $56.361,32$ \\
\hline & $\begin{array}{l}\text { Arzneimittel } \\
(n=26)\end{array}$ & $24.667,22$ & $4.512,14$ & 90 & $477.926,40$ \\
\hline & $\begin{array}{l}\text { Behandlungsmethoden } \\
\qquad(\mathrm{n}=98)\end{array}$ & $15.861,55$ & $5.873,47$ & 66,78 & $293.792,20$ \\
\hline & Gesamt & $13.276,83$ & $3.611,07$ & 66,78 & $477.926,40$ \\
\hline
\end{tabular}

Auch die Kategorisierung der Klagen nach Indikationsgruppen zeigt wesentliche Unterschiede hinsichtlich des Anteils der Rechtsprechungen, bei denen die Krankenkasse die Kosten zu erstatten hatte. Im Datensatz sind 62 Klagen zu Tumorerkrankungen enthalten, von denen 14 zu einer Kostenübernahmeverpflichtung führten (23\%). Prozentual am häufigsten wurde eine Erstattungsverpflichtung bei Erkrankungen der Augen und Ohren (56\%, $\mathrm{n}=28)$ sowie bei Erkrankungen des Bewegungsapparates beschieden $(54 \%, n=14)$. Keine der Klagen zu Erkrankungen des Mund- und Rachenraumes oder zu Schädeldeformitäten bei Säuglingen führte zu einer 
Erstattungsverpflichtung. Auch im Bereich ,Künstliche Befruchtung` ist die Rate mit einer von acht Rechtsprechungen (13\%) niedrig. Abbildung 2 stellt die Verteilung der absoluten Häufigkeiten der Rechtsprechungen in den einzelnen Indikationsgruppen gemeinsam mit dem Anteil jener Klagen dar, bei denen eine Erstattungsverpflichtung beschieden wurde.

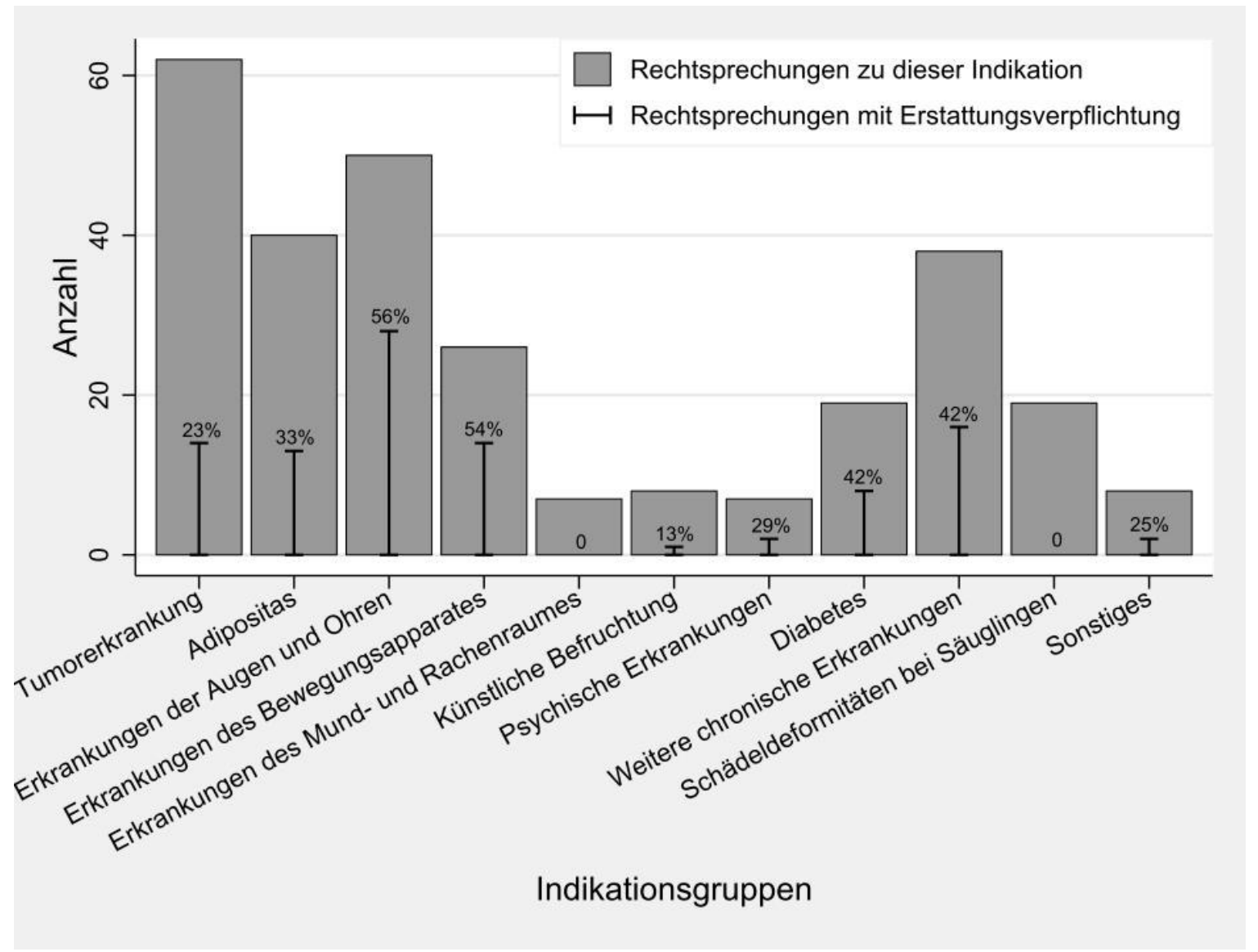

Abbildung 2. Rechtsprechungen je Indikationsgruppe und Anteil derer mit Erstattungsverpflichtung $(n=284)$

\section{Einzelbetrachtung von vier Technologien}

Einige Technologien treten in den Rechtsprechungen wiederholt auf. Anhand der vier am häufigsten eingeklagten Technologien wurde im Folgenden analysiert, inwiefern die juristischen Entscheidungen hinsichtlich der Kostenerstattung der Technologien über die Jahre variieren. Zusätzlich werden diese Entscheidungen dem Stand laufender oder abgeschlossener Bewertungen des G-BA gegenübergestellt. Abbildung 3 gibt einen 
Überblick hinsichtlich der beschiedenen Erstattungsverpflichtungen pro Jahr für die vier am häufigsten eingeklagten Technologien.
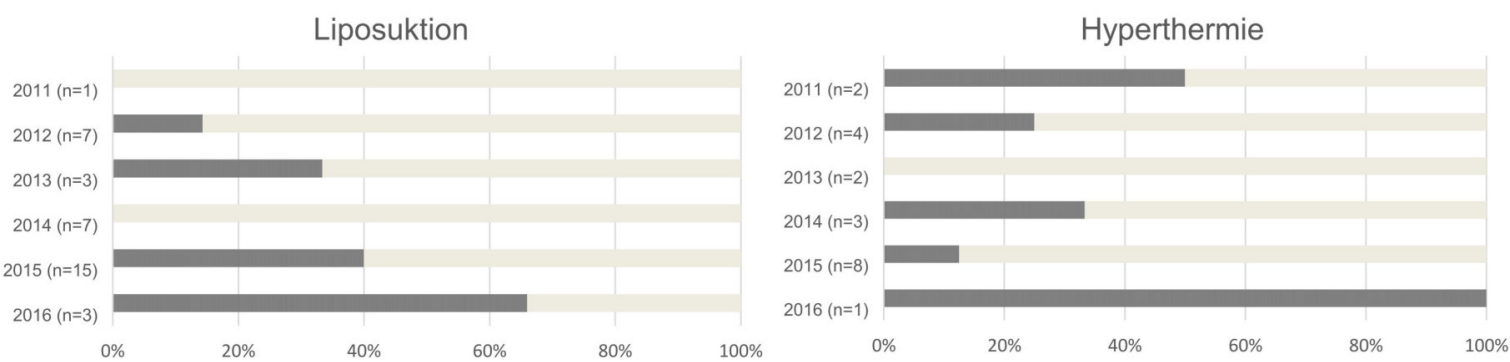

kontin. Glukosemessung
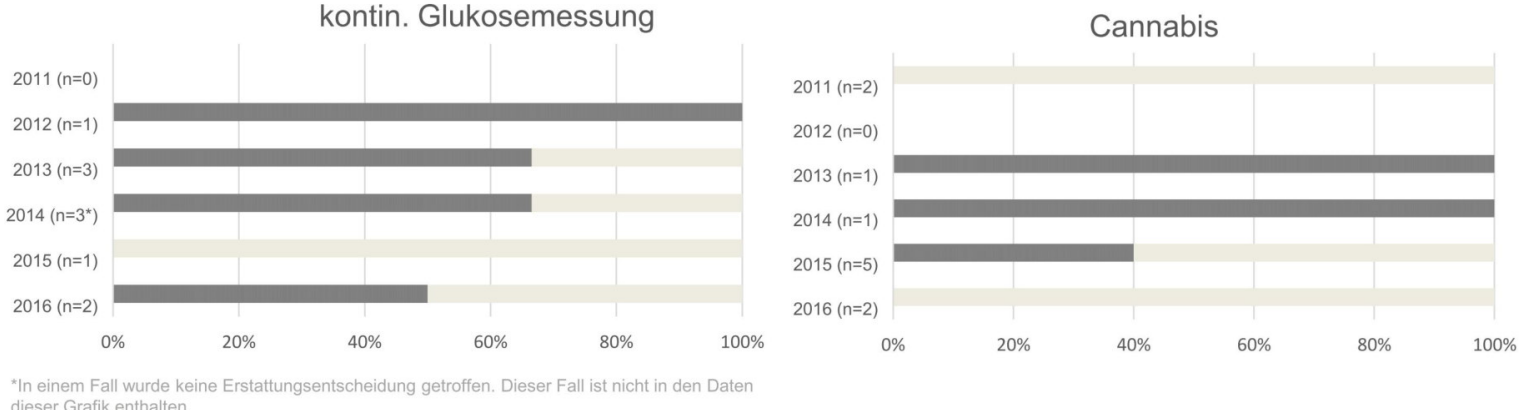

- Anteil Entscheidungen mit Erstattungsverpflichtung

Abbildung 3. Anteil der Rechtsprechungen mit und ohne Erstattungsverpflichtung pro Jahr der vier häufigsten Technologien $(n=78)^{7}$

Es liegen 36 Rechtsprechungen zur Liposuktion als Verfahren der Fettabsaugung bei Adipositas vor. Dabei fällt auf, dass auch bei Gerichtsverfahren mit inhaltlicher und zeitlicher Nähe, Rechtsprechungen häufig zu unterschiedlichen Entscheidungen hinsichtlich einer Erstattungsverpflichtung kamen. So wurde im März 2012 einer 34jährigen Klägerin in Sachsen die Durchführung einer ambulanten Liposuktion der Oberschenkel erstattet [15]. Hingegen wurde einen Monat später einer 35-jährigen Klägerin in Rheinland-Pfalz eine Liposuktion der Oberschenkel und Knieinnenseiten verwehrt [16]. Diese hatte die Behandlung in Anspruch genommen, ohne die Entscheidung der Krankenkasse abzuwarten. Im Jahr 2014 wurde auf Antrag der Patientenvertretung das Beratungsverfahren zur Bewertung der Liposuktion bei Lipödem beim G-BA eingeleitet. Das Ergebnis der Nutzenbewertung entscheidet darüber, ob die

\footnotetext{
${ }^{7}$ Das Jahr 2016 ist durch Ende des Beobachtungszeitraumes am 01.08.2016 nicht vollständig abgebildet.
} 
Methode künftig ambulant und stationär zulasten der GKV angewendet werden kann. Bis Juni 2016 waren betroffene Hersteller aufgefordert, sich mit aussagekräftigen Unterlagen zu melden, damit der G-BA das Stellungnahmerecht prüfen kann [17]. Das Verfahren läuft zum jetzigen Zeitpunkt.

Es wurden 20 Gerichtsverfahren zur Hyperthermie als Tumorbehandlung geführt. Davon beziehen sich vier Behandlungen auf den stationären Sektor, von denen eine anschließend erstattet wurde, und 16 auf den ambulanten Sektor, von denen vier erstattet wurden. Bereits im Jahr 2001 hatte der AOK-Bundesverband beim Vorgängergremium des G-BA die Bewertung der Hyperthermie beantragt. Der G-BA leitete noch 2001 das Beratungsverfahren ein. Im Mai 2005 wurde die Methode für die vertragsärztliche Versorgung ausgeschlossen [18].

Der Datensatz enthält elf Klagen zur kontinuierlichen Glukosemessung (Continuous Glucose Monitoring, CGM). Insbesondere in einer Rechtsprechung des BSG besteht zwischen den Beteiligten Uneinigkeit darüber, ob es sich bei CGM um ein Hilfsmittel oder um eine Behandlungsmethode handelt [19]. Bei der Betrachtung über die Jahre fällt auf, dass in der Rechtsprechung aus dem Jahr 2012 die Krankenkasse zur Kostenerstattung von CGM verpflichtet wird; Gleiches gilt für einen Großteil der Rechtsprechungen aus den Jahren 2013 und 2014. Hingegen werden Krankenkassen im Jahr 2015 und 2016 nur in einem Fall wegen Fristablauf der Antragsbearbeitung der Krankenkasse zur Erstattung verpflichtet, in allen anderen Fällen muss CGM hier nicht erstattet werden. Im Jahr 2011 hatte der GKV-Spitzenverband die Bewertung der kontinuierlichen interstitiellen Glukosemessung mit Real-Time-Messegeräten beantragt. Der G-BA leitete noch in 2011 das Beratungsverfahren ein. Im Juni 2016 wurde die Methode für die vertragsärztliche Versorgung für bestimmte Indikationen und mit Vorgaben zur Qualitätssicherung zugelassen.

Bei den elf Klagen zur Behandlung mit Cannabis ist ein solcher kontinuierlicher Trend nicht zu beobachten. Aus den Klagen 2011 resultierte keine Erstattungsverpflichtung, 
jedoch musste die gleiche Therapie 2013 und 2014 in allen Fällen und 2015 vereinzelt von der GKV erstattet werden, 2016 wurde erneut keine Erstattungsverpflichtung ausgesprochen. Von den elf Rechtsprechungen beziehen sich sieben auf den Wirkstoff Dronabinol und vier auf die Anwendung von Medizinal-Cannabisblüten. Die Rechtsprechungen beziehen sich auf verschiedene Indikationen (bspw. Spastik und Ovarialkarzinom).

\section{Nikolausurteil}

Eine Sonderregelung zur Anwendung von neuen Gesundheitstechnologien ist deren Einsatz bei lebensbedrohlichen oder regelmäßig tödlich verlaufenden Erkrankungen. Das Nikolausurteil (bzw. § 2 Abs. 1a SGB V) findet im Datensatz in rund 40 Klagen Anwendung. Die genaue Anzahl ist ggf. höher, da das Nikolausurteil nicht immer in der Begründung zur Erstattung angeführt wird. Ein Beispiel für eine Rechtsprechung gemäß Nikolausurteil ist die erfolgreich eingeklagte Erstattung einer allogenen Stammzellentransplantation bei einer Patientin mit akuter Leukämie. Die Begründung, mit der die Krankenkasse vorab eine Kostenerstattung abgelehnt hatte, war eine nicht ausreichende Erprobung der Behandlung für Patienten im Alter der Klägerin [20]. Ein weiteres Beispiel betrifft eine Patientin mit inoperablem fortgeschrittenem Magenkarzinom mit Metastasen in mehreren Organen und einer Überlebensprognose von sechs bis zwölf Monaten. In diesem Fall wurde die Krankenkasse zur Erstattung einer Stopflow-Chemotherapie [21] verpflichtet.

Auch bei Rechtsprechungen gemäß Nikolausurteil wurden in Klagen zur gleichen Therapie uneinheitliche Urteile gefällt. So klagte eine Klägerin die Kostenerstattung der Behandlung mit dendritischen Zellen am LSG Baden-Württemberg im März 2014 erfolgreich ein [22]. Ebenfalls im März 2014 lehnte das LSG Schleswig-Holstein die Erstattung einer solchen Behandlung mit der Begründung ab, diese befänden sich in der „wissenschaftlichen Entwicklung“, sodass ihre Anwendung „außerhalb von kontrollierten klinischen Studien“ nicht zu empfehlen sei [23]. Ein weiterer Streitgegenstand war die 
Auffassung, wann alternative Therapiemöglichkeiten ausgeschöpft seien. So wird einer Patientin mit Mammakarzinom die Erstattung einer brusterhaltenden Therapie mit Verweis auf die Möglichkeit einer operativen Entfernung der Brust abgelehnt [24].

\section{Diskussion}

Durch die Analyse der Rechtsprechungen werden Tendenzen identifiziert, in welchen Konstellationen (Technologie, Indikation, Sektor etc.), Krankenkassen zur Kostenerstattung neuer Technologien verpflichtet wurden. In der Analyse wird deutlich, dass für den stationären Sektor die Anzahl eingereichter Klagen niedriger ist als im ambulanten Sektor. Jedoch zeigen die Ergebnisse auch, dass die Krankenkassen in 40 Prozent der Rechtsprechungen für den stationären Sektor verpflichtet wurden (verglichen mit 33\% im ambulanten Sektor), die Kosten zu erstatten. Eine Erklärung dafür kann im Verbotsvorbehalt gesehen werden. Damit werden prinzipiell alle Leistungen erstattet, die nicht vom G-BA ausgeschlossen sind, sodass zunächst mehr Leistungen im Krankenhaus erstattungsfähig sind als im ambulanten Sektor und davon auszugehen ist, dass damit weniger Klagen gestellt werden. Während prinzipiell alle Leistungen zu erstatten sind, müssen diese trotzdem das Qualitäts- und Wirtschaftlichkeitsgebot erfüllen (§ 2 Abs. 1 und § 12 SGB V). Die Einhaltung obliegt zunächst dem Krankenhaus bzw. dem behandelnden Arzt. Für behandelnde Ärzte bzw. Krankenhäuser kann demnach Unsicherheit bestehen, welche neuen Leistungen erstattet werden [25]. Dazu führen Huster und Trefz an, dass die Entscheidungsmacht des G-BA in Urteilen des BSG über die Zeit unterschiedlich ausgelegt wurde $[26,27]$. Bis 2008 ging die ständige Rechtsprechung von einem Entscheidungsmonopol des G-BA aus. Demnach durften Krankenhäuser jegliche Gesundheitstechnologien, die nicht vom G-BA ausgeschlossen waren, unter Einhaltung der Wirtschaftlichkeit anwenden. Seit dem Urteil des BSG vom 28.07.2008 [28] wird der Verbotsvorbehalt hingegen so ausgelegt, dass auch neue Gesundheitstechnologien dem ,allgemein anerkannten Stand der medizinischen Erkenntnisse‘ entsprechen müssen. Die Möglichkeit der Einzelfallüberprüfung neben einer Bewertung durch den G-BA wurde damit gestärkt. Die Neuauslegung des 
Verbotsvorbehalts hat damit zu einer gestiegenen Unsicherheit hinsichtlich der Finanzierung geführt. Aufgrund des Zeithorizonts der hier analysierten Fälle (2011-2016) ist es jedoch nicht möglich, diesen Aspekt tiefergreifend zu untersuchen. Es liegt jedoch nahe, dass mit diesem Urteil die Fälle der Klagen für stationäre Leistungen gestiegen sind.

Auf Grundlage der Ergebnisse sollte überlegt werden, ob die Vorgaben, welche Leistungen im stationären Sektor unter welchen Bedingungen und durch wen zu erstatten sind, durch den Gesetzgeber konkretisiert werden sollten. Hierbei stellt die Einführung einer Bewertung von Methoden unter Anwendung von Hochrisiko-Medizinprodukten (§ 137h SGB V) einen ersten Ansatz dar. Damit werden bestimmte Medizinprodukte einer Nutzenbewertung unterzogen, um zusätzliche Evidenz für die Einführung in die Regelversorgung zu generieren (sogenannte „Coverage with Evidence Development“). Während dieser Phase wird die Behandlung durch die Krankenkassen erstattet. Mittelfristig sollte eine solche Evidenzgenerierung auch auf weitere Technologien ausgeweitet werden, da diese Regelung nur gilt, wenn vorab ein gesondertes Entgelt (sog. NUB-Entgelt, § 6 Abs. 2 KHEntG) beantragt wurde [29]. Mit dieser zusätzlichen Evidenz könnten Einzelfallentscheidungen zukünftig verringert werden bzw. nach klaren Maßstäben durch die Gerichte entschieden werden. Dieser Ansatz würde zudem das Prinzip der evidenzbasierten Medizin stärken, nach der Entscheidungen, die ansonsten von Mehrheiten und Interessen geleitet werden, auf Grundlage von (wissenschaftlicher) Evidenz getroffen werden sollen.

Bei Betrachtung der Rechtsprechungen zu Medizinprodukten im ambulanten Sektor betreffen 89 Prozent Hilfsmittel. Bei diesen wird deutlich häufiger eine Erstattungsverpflichtung für die Krankenkassen festgelegt als für die übrigen Technologien in der ambulanten Versorgung. Hilfsmittel werden im deutschen Gesundheitswesen erstattet, sofern sie im Hilfsmittelverzeichnis gelistet sind. Da dieses keine ausschließliche Positivliste darstellt, können auch innovative Technologien Anwendung finden, die noch nicht darin aufgenommen sind. Das könnte den hohen 
Anteil an Klagen zu Hilfsmitteln erklären. Eine weitere Problematik liegt gemäß Rechtsprechungen in der Zuordnung eines Produktes zur Technologiegruppe (z. B. Hilfsmittel vs. Behandlungsmethode), die in Einzelfällen nicht eindeutig ist. Die Rahmenbedingungen zur Erstattung neuer Hilfsmittel (Aufnahme in das Hilfsmittelverzeichnis durch den GKV-Spitzenverband) unterscheiden sich zu denen der Behandlungsmethoden (Methodenbewertung durch den G-BA), einschließlich anderer Ansprechpartner, Entscheidungskriterien und Verfahrensdauern. Wenn mangels klarer Abgrenzungen die Zuordnung einer Gesundheitstechnologie zum Bereich der Hilfsmittel oder der Behandlungsmethoden unsicher ist, wie bei der kontinuierlichen Glukosemessung, kann dies zu einer massiven Unsicherheit hinsichtlich der Anwendung durch Ärzte in der Versorgung und der Erstattungspflicht führen. So wurde die CGM über die Jahre mit abnehmender Tendenz erstattet, bis sie 2015 und 2016 von den Sozialgerichten vollständig, mit Ausnahme einer Fristversäumnis, abgelehnt wurden. Während dieser Zeit lief eine Methodenbewertung beim G-BA (auf Antrag des GKVSpitzenverbands im Jahr 2011), wodurch die CGM im September 2016 in den Leistungskatalog der GKV aufgenommen wurde [7]. Es ist auf Grundlage der Ergebnisse denkbar, dass neue Technologien aktiv von einem Eintritt in die Versorgung behindert werden, wenn nicht klar ist, ob die Aufnahme einer Technologie in den Leistungskatalog über den Hilfsmittelkatalog oder die Methodenbewertung erfolgt.

Die Analyse der Rechtsprechungen der vier Technologien zeigt inkonsistente Entscheidungen hinsichtlich der Kostenerstattung bei sehr ähnlichen Fällen. Es sind keine Muster erkennbar, dass die Verpflichtung zur Kostenerstattung der gleichen Technologie über mehrere Jahre kontinuierlich zu- oder abnehmen würde: Dabei ist für Patienten besonders problematisch, wenn Methodenbewertungen erst nach vielen Jahren der Anwendung, Einzelfallabrechnung sowie Klagen aufgenommen und nach weiteren mehreren Jahren entschieden werden. Bei der Liposuktion wurde nach elf beschiedenen Klagen im Mai 2014 eine Methodenbewertung beim G-BA eingeleitet. Zwischenzeitlich wurden bis Ende des Beobachtungszeitraums weitere 25 
Rechtsprechungen beschieden, während ein Beschlusszeitpunkt der

Methodenbewertung nicht ersichtlich ist. Ein Grund für die unterschiedlichen

Entscheidungen zur Erstattung könnte eine mangelnde Datengrundlage zum Nutzen der Technologien sein. Mit Einführung der Erprobungsregelung im Rahmen der Methodenbewertung des G-BA im Januar 2012 (§ 137e SGB V) wurde das Ziel verfolgt, neue Technologien mit potenziell vorliegendem Nutzen zu erstatten, während weitere Evidenz zum Nutzen in Studien ermittelt wird [30]. Damit besteht für Patienten die Möglichkeit die Leistung zu erhalten und auch erstattet zu bekommen. Das Verfahren erweist sich bislang jedoch als sehr träge, da bis zum jetzigen Zeitpunkt (Juli 2017) erst zwei Richtlinien zur Erprobung beschlossen wurden [31,32]. Für eine Entscheidung hinsichtlich der Erstattungsfähigkeit wäre es daher vorteilhaft, gesetzliche Nutzenbewertungen frühzeitig und mit zeitlichen Fristen durchzuführen und die Erprobungsregelung weiter zu stärken.

Bei Klagen zu Behandlungen lebensbedrohlich erkrankter Patienten warf die Umsetzung des Nikolausurteils die Fragen auf, wann keine medizinischen Alternativen vorhanden sind und welche Nutzennachweise für eine Kostenerstattung in diesem Rahmen notwendig sind. Aufgrund dieser Problematik wurden zahlreiche Therapien trotz Nikolausurteil nicht erstattet. Die Tatsache, dass der Gesetzgeber dieses höchstrichterliche Urteil mit dem Versorgungsstrukturgesetz ins Sozialgesetzbuch aufgenommen hat, spricht dafür, dass die Zusage des Nikolausurteils weiter gestärkt werden sollte. Die Ergebnisse werfen damit die Frage auf, ob die Intentionen des Gesetzgebers hier unzureichend umgesetzt werden.

\section{Limitationen}

Rechtsprechungen in Deutschland betreffen - mit Ausnahme höchstrichterlicher Urteile den Einzeltatbestand [33]. Dementsprechend muss eine Rechtsprechung nicht anderen Rechtsprechungen zu derselben Technologie folgen, sondern kann zu einer unterschiedlichen Entscheidung hinsichtlich der Erstattung kommen. Trotzdem bietet 
diese Analyse der gerichtlichen Einzelfallentscheidungen einen ersten und wichtigen Einblick in den Zugang von Patienten zu neuen Gesundheitstechnologien, die (noch) nicht in den Leistungskatalog aufgenommen sind. Die Erstattungsentscheidung in Rechtsprechungen hängt neben inhaltlichen Gründen teilweise von formellen Verfahrensabläufen ab, wie der gesetzlichen Antwortfrist von Krankenkassen bei Leistungsanträgen (§ 13 Abs. 3a SGB V), die in der vorliegenden Studie nicht berücksichtigt sind. Auch kann nicht analysiert werden, wie häufig Einzelfallanträgen beim ersten Antrag stattgegeben oder widersprochen wurde. Dies wird nicht systematisch erfasst und ist entsprechend nur durch Krankenkassen selbst nachvollziehbar. Es werden Unterschiede zwischen den betrachteten Rechtsprechungen aufgezeigt, jedoch lassen die teilweise geringen Fallzahlen an Fallbeispielen insgesamt keine Rückschlüsse auf verallgemeinerbare Aussagen zu. Zudem unterscheidet sich in der Datenbank juris das Entscheidungsdatum der Rechtsprechung vom Zeitpunkt des Einstellens. Dokumente, die nach dem Zeitpunkt der letzten Recherche für den Beobachtungszeitraum eingepflegt wurden, fanden in der Analyse keine Berücksichtigung. Trotz Limitationen der Studie gibt diese erstmalig Einblicke in richterliche Entscheidungen zu Einzelfallanträgen und zeigt den Patientenzugang über Einzelfallanträge, die per Gericht entschieden werden.

\section{Fazit}

Die Studie gibt einen Überblick über und ersten Einblick in den Zugang von Patienten zu neuen Gesundheitstechnologien, bei denen ein Einzelfallantrag bei Krankenkassen abgelehnt und Klagen zur Erstattung eingereicht worden waren. Dabei werden einige Schwierigkeiten in der Praxis der Kostenerstattung von Gesundheitstechnologien identifiziert: Rechtsprechungen zu gleicher Technologie, gleichem Sektor und ähnlichem Zeitpunkt führen zu unterschiedlichen Ergebnissen hinsichtlich der Erstattung. Damit ist anzunehmen, dass die Ergebnisse aus Patientenperspektive in Teilen willkürlich erscheinen. Die Rechtsprechungen zu neuen Technologien stehen teilweise im Widerspruch zu darauffolgenden Entscheidungen der G-BA Methodenbewertung, was 
möglicherweise an zusätzlicher Evidenz über die Jahre liegen kann. Häufig liegen jedoch nach Jahren verbreiteter Anwendung einer Technologie kaum Studien zum Nutzen vor. Unabhängig von der Kostenerstattung durch das Solidarsystem setzen Patienten damit Hoffnung in ungewisse Behandlungen mit neuen Technologien. Für versorgende Ärzte ist eine wirtschaftliche Unsicherheit bei der Anwendung neuer Technologien anzunehmen. Im Gegensatz dazu verfolgt das deutsche Gesundheitssystem bzw. der Gesetzgeber die Grundsätze der evidenzbasierten Medizin. Die Ergebnisse dieser Studie weisen jedoch darauf hin, dass mehr Wissen über den Nutzen von neuen Technologien notwendig ist, um eine evidenzbasierte medizinische Versorgung zu ermöglichen. Der Generierung zusätzlicher Evidenz muss daher eine große Rolle zugeschrieben werden. Diese Ziele können u. a. über eine Konkretisierung der gesetzlichen Vorgaben zum Verbotsvorbehalt, eine Verpflichtung zur Studiendurchführung nach der Marktzulassung, um kontinuierlich zusätzliche Evidenz zu generieren, sowie eine schnellere Bewertung des Nutzens von neuen Technologien durch die gesetzlich beauftragten Institutionen verfolgt werden. Erste Ansätze sind mit der Erprobungsregelung vorhanden.

Diese Studie ist ein erster Ansatz, die Rechtsprechungen zur Kostenerstattung von Gesundheitstechnologien zu untersuchen. Auf dieser Grundlage sollten weitere Untersuchungen einzelner Rechtsprechungen, z. B. einer spezifischen Technologie, durchgeführt werden, die detailliert die Begründungen zur Entscheidung analysieren und diese auf Konsistenz untereinander prüfen. 


\section{Literatur}

[1] Sorenson C, Drummond M. Improving Medical Device Regulation: The United States and Europe in Perspective. The Milbank Quarterly 2014;92(1):114-150.

[2] Sorenson C, Drummond M, Torbica A, Callea G, Mateus C. The role of hospital payments in the adoption of new medical technologies: an international survey of current practice. Health economics, policy, and law 2015;10(2):133-59.

[3] Basu S, Hassenplug JC. Patient access to medical devices--a comparison of U.S. and European review processes. The New England journal of medicine 2012;367(6):4858.

[4] Kreis J, Busse R. From evidence assessments to coverage decisions?: the case example of glinides in Germany. Health policy (Amsterdam, Netherlands) 2012;104(1):27-31.

[5] Bech M, Christiansen T, Dunham K, Lauridsen J, Lyttkens CH, McDonald K et al. The influence of economic incentives and regulatory factors on the adoption of treatment technologies: a case study of technologies used to treat heart attacks. Health economics 2009;18(10):1114-32.

[6] Neumann U, Hagen A, Schönermark M. Regulation der Aufnahme von innovativen nichtmedikamentösen Technologien in den Leistungskatalog solidarisch finanzierter Kostenträger; 2007.

[7] Gemeinsamer Bundesausschuss. Richtlinie Methoden verträgsärztliche Versorgung und Richtlinie Methoden Krankenhausbehandlung: der Kontinuierlichen interstitiellen Glukosemessung mit Real-Time Messgeräten zur Therapiesteuerung bei Patienten mit insulinpflichtigem Diabetes mellitus [accessed 26 November 2017]; Available from: https://www.g-ba.de/informationen/beschluesse/2623/.

[8] Gemeinsamer Bundesausschuss. Informationsblatt. Voraussetzungen der Erbringung einer (neuen) Methode zu Lasten der gesetzlichen Krankenversicherung (GKV) [accessed 13 July 2017]; Available from: https://www.g-ba.de/downloads/17-983563/Infoblatt_Voraussetzungen-Erbringung-Methode_2013-10-10.pdf. 
[9] OECD. New Health Technologies - Managing Access, Value and Sustainability. Paris: OECD Publishing; 2017.

[10] Institut für Qualität und Wirtschaftlichkeit im Gesundheitswesen. Allgemeine Methoden: Version 4.2. KöIn: Institut für Qualität und Wirtschaftlichkeit im Gesundheitswesen; 2015.

[11] Centre for Reviews and Dissemination. CRD's guidance for undertaking reviews in healthcare. 3. ed. York: York Publ. Services; 2009.

[12] Sorenson C, Kanavos P. Medical technology procurement in Europe: a crosscountry comparison of current practice and policy. Health policy (Amsterdam, Netherlands) 2011;100(1):43-50.

[13] LSG Baden-Württemberg. Urteil vom 16.07.2015 - Az. L 11 KR 211/15.

[14] SG Bremen. Urteil vom 05.07.2011 - Az. S 4 KR 15/06.

[15] SG Chemnitz. Urteil vom 01.03.2012 - Az. S 10 KR 189/10.

[16] SG Mainz. Urteil vom 23.04.2012 - Az. S 14 KR 143/11.

[17] Gemeinsamer Bundesausschuss. Bekanntmachung des Gemeinsamen Bundesausschusses über weitere Beratungsthemen zur Überprüfung gemäß $\S 135$ Abs. 1 und § 137c des Fünften Buches Sozialgesetzbuch (SGB V): Liposuktion bei Lipödem [accessed 26 November 2017]; Available from: https://www.gba.de/downloads/40-268-3175/2014-05-22_135-137c-

SN_Antragsannahme_Liposuktion-bei-Lip\%C3\%B6dem_BAnz.pdf.

[18] Bundesministerium für Gesundheit und Soziale Sicherung. Bekanntmachungen: Bekanntmachung eines Beschlusses des Gemeinsamen Bundesausschusses über eine Änderung der Anlage B "Nicht anerkannte Untersuchungs- und Behandlungsmethoden" der Richtlinie zur Bewertung medizinischer Untersuchungsund Behandlungsmethoden (BUB-Richtlinie) [accessed 17 July 2017]; Available from: https://www.g-ba.de/downloads/39-261-199/2005-01-18-BUB-Hyperthermie.pdf.

[19] BSG 3. Senat. Urteil vom 08.07.2015 - Az. B 3 KR 5/14 R.

[20] SG Hamburg. Urteil vom 17.01.2013 - Az. S 35 KR 118/10. 
[21] LSG Schleswig-Holstein. Urteil vom 08.07.2016 - Az. L 5 KR 105/16 B ER, L 5 AR.

[22] LSG Baden-Württemberg. Urteil vom 19.03.2014 - Az. L 5 KR 1496/13.

[23] LSG Schleswig-Holstein. Urteil vom 13.03.2014 - Az. L 5 KR 95/10.

[24] SG Freiburg (Breisgau). Urteil vom 26.07.2012 - Az. S 5 KR 5749/10.

[25] Baum G. Auswirkungen der BSG Rechtsprechung auf Einsatz und Vergütung Neuer Untersuchungs- und Behandlungsmethoden: (Schreiben vom 15.05.2014 an Ministerialdirektor Dr. Ulrich Orlowski) [accessed 30 March 2016]; Available from: http://www.dkgev.de/media/file/17331.Anlage2_NUB__Ablehnung_d._Leistungen_zur_renalen_Denervation_durch_d._GKV.pdf.

[26] Trefz U. NUB-Entgelte: Qualitätsgebot als Innovationsbremse? f\&w führen und wirtschaften im Krankenhaus;2014(05):496.

[27] Huster S. Krankenhausrecht und SGB V - Medizinische Innovationen im stationären Sektor. Gesundheitsrecht;2010(07):337-44.

[28] BSG 1. Senat. Urteil vom 28.07.2008 - Az. B 1 KR 5/08 R.

[29] Ex P, Busse R, Henschke C. Die Nutzenbewertung von nichtmedikamentösen Untersuchungs- und Behandlungsmethoden: Welche Tragweite hat die Regelung nach § 137h SGB V? Gesundheit- und Sozialpolitik 2016;70(2):48-55.

[30] Olberg B, Perleth $M$, Busse $R$. The new regulation to investigate potentially beneficial diagnostic and therapeutic methods in Germany: up to international standard? Health policy (Amsterdam, Netherlands) 2014;117(2):135-45. 10.1016/j.healthpol.2014.04.018.

[31] Gemeinsamer Bundesausschuss. Beschluss des Gemeinsamen Bundesausschusses über eine Richtlinie zur Erprobung der Magnetresonanztomographie-gesteuerten hochfokussierten Ultraschalltherapie zur Behandlung des Uterusmyoms [accessed 17 July 2017]; Available from: https://www.g-ba.de/downloads/39-261-2810/2016-12-15_Erp-RL_MRgFUSTUF_BAnz.pdf. 
[32] Gemeinsamer Bundesausschuss. Beschluss des Gemeinsamen Bundesausschusses über eine Richtlinie zur Erprobung der allogenen Stammzelltransplantation bei Multiplem Myelom jenseits der Erstlinientherapie [accessed 17 July 2017]; Available from: https://www.g-ba.de/downloads/39-2612841/2017-01-19_Erp-RL_SZT_MM_Multiples-Myelom_BAnz.pdf.

[33] Krasney OE. Die Bedeutung höchstrichterlicher Urteile für die ärztliche Begutachtung. Praktische Arbeitsmedizin, S. 6-7.

[34] Transparent Reporting of Systematic Reviews and Meta-Analyses. PRISMA Flow Diagram [accessed 24 October 2016]; Available from: http://prismastatement.org/PRISMAStatement/FlowDiagram.aspx. 


\section{Legenden}

\section{Abbildung 1}

PRISMA-Diagramm: Ergebnisse der Recherche und des Selektionsprozesses (nach dem Modell des Centre for Reviews and Dissemination sowie des Instituts für Qualität und Wirtschaftlichkeit im Gesundheitswesen) [34].

\section{Tabelle 1}

Erstattungsverpflichtung neuer Gesundheitstechnologien in Rechtsprechungen je Technologiegruppe, getrennt nach Sektoren

\section{Tabelle 2}

Von Klägern geforderter Preis der Technologie in Euro, nach Technologiegruppen gegliedert

\section{Abbildung 2}

Rechtsprechungen je Indikationsgruppe und Anteil derer mit Erstattungsverpflichtung $(n=284)$

\section{Abbildung 3}

Anteil der Rechtsprechungen mit und ohne Erstattungsverpflichtung pro Jahr der vier häufigsten Technologien $(n=78)^{8}$

\footnotetext{
${ }^{8}$ Das Jahr 2016 ist durch Ende des Beobachtungszeitraumes am 01.08.2016 nicht vollständig abgebildet.
} 